\title{
Influence study of concentrated photovoltaic location on voltage stability
}

\author{
Erhab Youssef *, Rasha M. El Azab, Amr M. Amin \\ Department of Electrical Power Engineering, Faculty of Engineering, Helwan University, Cairo, 11792, Egypt \\ Academy of Scientific Research and Technology, Al-Kasr El-Aynay, Cairo, 11516, Egypt
}

\begin{abstract}
Concentrated Photovoltaic modules (CPV) use optics, such as lenses to concentrate a large amount of sunlight onto a small area of solar photovoltaic materials to generate electricity. The solar irradiance level and landscape area are considered as main factors in CPV site on the network. These factors are not sufficient for improving the CPV performance and grid integration . The coupling point of CPV to the grid is an important role and affects the network stability. This paper focuses on the influence study of CPV location on voltage stability .PSS/E has been used as a tool for simulation and validation the integration of CPV to 14-IEEE network as benchmark test. Ac contingency analysis tool is used to study the voltage stability at different cases and locations of CPV. Results indicate that the distributed CPV improves the voltage stability of the network, rather than centralized CPV. Distributed CPV connects at different locations and near to the load. Therefore, the impact of distributed CPV is minimized with respect to voltage stability issue.
\end{abstract}

Keywords:CPV, contingency analysis, PSS/E, voltage stabilityand location

\section{Introduction}

CPV is a photovoltaic technology that generates electricity from sunlight. Contrary to conventional photovoltaic systems, it uses lenses and curved mirrors to focus sunlight onto small, but highly efficient, Multi-Junction (MJ) solar cells. In addition, CPV systems often use solar trackers and sometimes a cooling system to further increase their efficiency [1], [2]. On-going research and development is rapidly improving their competitiveness in the utility-scale segment and in areas of high solar insolation.

The integration of renewable energy (especially wind and solar) into the existing power systems is one of the main challenges due to the major concerns about the power system stability as well as system reliability. In the power system environment, the voltage stability is one of the main indicators of the power system stability [3].

In general the voltage stability problems occur more frequently in a heavily loaded system. The change in voltage is directly proportional to the change in load. Hence, voltage stability is sometimes termed as load stability [4].

Among stability issues, voltage instability has been a major concern for power system. Several major power interruptions have been linked to power system voltage instability in recent past [5]. It has been proved that inadequate reactive power compensation during stressed operating condition can lead to voltage instability.

The integration of CPV to grid has two types, as follows: First, grid-connected distributed that Provide power to a number of grid-connected customers on their premises or directly to the grid. It has many features, such as it can be integrated into the customer's premises to increase reliability and reduce dependency on the grid. It also plays a role in the smart grid management. Second, Grid-connected

\footnotetext{
* Manuscript received May 8, 2015; revised August 15, 2015.

Corresponding author. Tel.:+2018753451 E-mail address: erhabYoussef@h-eng.helwan.edu.eg.

doi: $10.12720 /$ sgce.4.3.226-232
} 
centralized CPV plants, which provide bulk power as a centralized power station. Its main features are oil independence and reduction in green-house-gases with minimum operation and maintenance expenditure [6]. CPV generator in medium and long terms, as recent studies suggested, will become very attractive commercially. Also, large-scale implementations of CPV plant are implemented in many parts of the world [7].

Recently, the increasing penetration levels of CPV plants are raising concerns to utilities due to possible negative impacts on power system stability as speculated by a number of studies [8]. Thus, the thorough investigation of power system stability with large-scale PV is an urgent task.

However, there are some related technical concerns that should be addressed beforehand CPV generation. The characteristics of these generators are inherently different from those of traditional generators. Integrating these resources into the bulk power system shouldn't jeopardize the grid reliability and security [9].

Although large-scale PV is capable of generating reactive power, however, the operation of PV in terminal voltage mode has the potential for adverse interaction with other voltage controllers [9].

Therefore, grid code requires operation at power factors equal or greater than 0.95 for PV generators, [10] [11].Moreover, the size and position of large-scale PV generator can introduce detrimental effect on power system voltage stability as the level of PV penetration increased.

In this paper, the controller types of CPV generators are demonstrated and discussed in details. The voltage stability analysis is used to study the performance of integration CPV introduce the paper, with a large scale into grid. Influence of location CPV is studied with different cases. The IEEE-14 bus is used as benchmark with integration of CPV.

This paper is structured as follows: Section I, presents the proposed model and discusses in details. Section II, shows the system description of the integration of CPV to IEEE-14 bus as benchmark. Section III, presents the simulation results. Finally, the main conclusions and contributions of the paper are highlighted in Section IV.

\section{System Description}

This section presents the typical structure of a grid connected CPV and the main controller types for $\mathrm{CPV}$ are discussed. PSS/E program is used to develop the CPV generator models for simulation.

\subsection{System modeling}

The schematic diagram of a grid connected PV generator is depicted in Fig. 1. It consists of PV module, dc/dc converter, dc/ac inverter and coupling transformer. A storage system is in general absent in large grid-connected PV installations, except for small critical loads of the plant such as start-up controls. However, there are some instances in which considerable storage has been integrated into large scale PV [12].

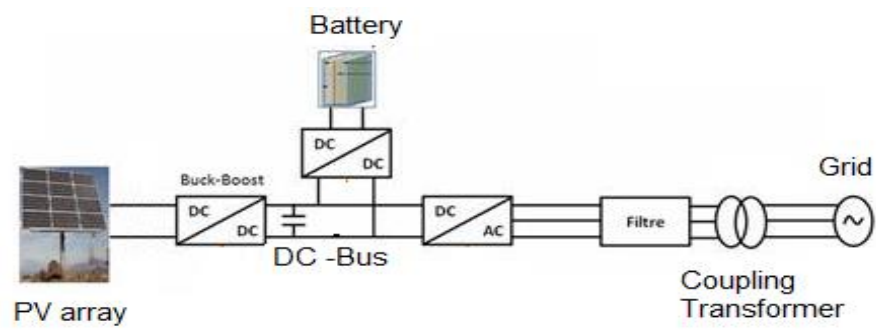

Fig. 1. A typical structure of a grid connected PV generator.

The task of tracking the Maximum Power Point (MPPT) in PV is usually performed by a DC/DC converter at the output of the array, which regulates the voltage to the desired value. Since no moving parts are employed in this process, the response of the MPPT can be considered instantaneous for system 
stability studies [13], [14].

The power inverter module calculates the current injection to the grid based on filtered active and reactive power commands from the electrical control module. The inverter control module includes reactive and active power controls [15].

The reactive control calculates the reactive current command for the remote bus voltage control. The active power control compares the active power injected to the grid against the power reference, and changes the active component of the injected current respectively as depicted in Fig. 2. The power reference is controlled by the amount of DC power coming from the panel module.

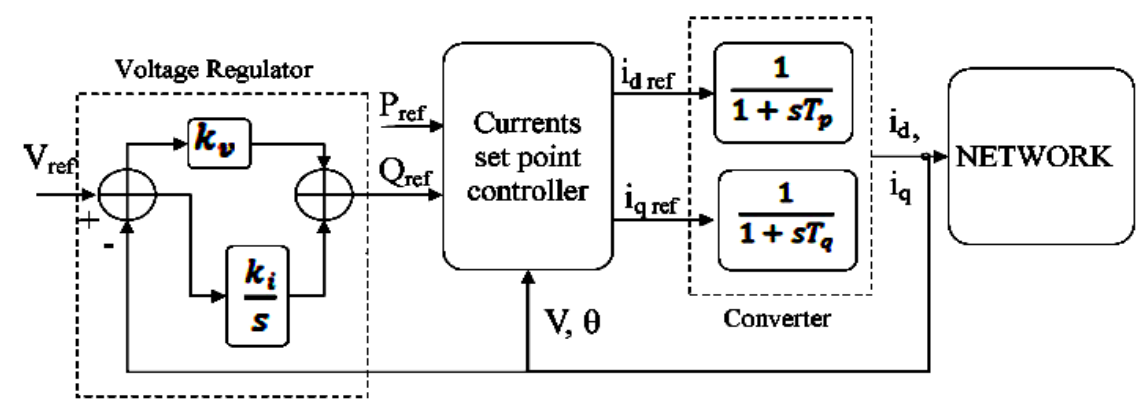

Fig. 2. Simplified control block of inverter model of PV.

The PSS®E Solar PV Unit dynamic stability model was developed to simulate the performance of a PV plant connected to the grid via a power converter. The model is largely based on the generic wind turbine model type 4 (WT4). This type represents the induction generator connected to the gird via full scale power electronics [16].

\section{Numerical Studies}

Static analysis used to study the performance of a large scale PV connected to grid. CPV and distributed PV are simulated.All numerical studies were carried out by PSS/E program.

\subsection{System description}

IEEE 14-bus network consists of two synchronous generators at bus 1 and 2, and three synchronous condensers at bus 3, 8 and 6 as shown in Fig. 3. The technical data of the IEEE 14-bus is found in [17]. Total loads of the network are 310.8 MW and 88.2 MVAR with increasing by $20 \%$ from base load data of the network. All loads are considered as constant P and constant Q, as a most severe load model case for stability studies. Aggregation model is used to develop CPV generators.

In this section, two cases are developed to study the influence of location of CPV and distributed PV. The penetration level of PV in this study is $30 \%$.

Voltage stability analysis tools are studied and discussed in details in [18]. In this paper, AC contingency is used to study the voltage stability by monitoring voltage buses at certain events or contingencies.

\subsection{Simulation results}

- IEEE-14 bus without renewable energy (RE):

AC contingency analysis is applied to the IEEE 14-bus network as a base case to define critical buses. Table 1 shows the worst contingency and their related voltages with less than $0.95 \mathrm{pu}$ and more than 1.05 pu. 


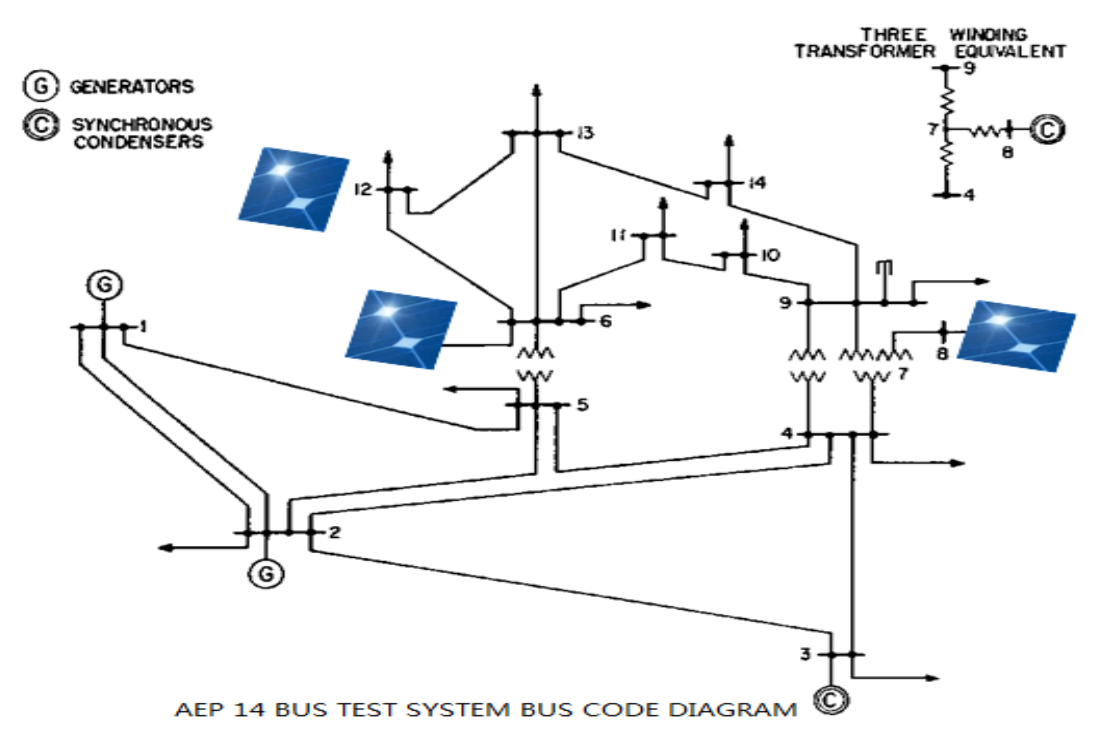

Fig. 3. Locations of CPV at IEEE-14 bus network.

Table 1. Voltage buses at worst contingencies

\begin{tabular}{lcl}
\hline Worst Contingency & Bus No: & Max Voltage Violation (pu) \\
\hline & Voltage with less than 0.95 \\
Single 2-3 (1) & 3 & 0.89516 \\
Single 5-6 (1) & 6 & 0.94721 \\
Single 5-6 (1) & 12 & 0.93158 \\
Single 5-6 (1) & 13 & 0.93318 \\
Single 5-6 (1) & 14 & 0.94881 \\
\hline
\end{tabular}

As listed in Table 1, the outage line from (5-6) and line (2-3) are the worst critical contingencies Most of the voltages buses are less than 0.95 at this event . These buses are 12,13,6 and 14 in order.

From this table, at outage line from bus 5 to bus 6 , the weakest bus is 12 and bus 6 and 14 is the critical stable. Bus 3 has voltage less than 0.95 that is 0.89156 at outage line (2-3).

Therefore, the CPV is connected at (bus 6,8 and 12) for each case and study the impact of location on voltage stability at the outage of line (5-6) and line (2-3).

The distributed PV is connected to buses $(12,14)$, buses $(6,13)$ and buses $(8,10)$ at each case. These buses are estimated based on the recorded results in Table I with take in consideration load demand at these buses. These cases are demonstrated as follow:

- IEEE-14 bus with CPV generators:

In this study the penetration level of CPV is $30 \%$ of the generated power .The sizing of PV is $80 \mathrm{MW}$. CPV generates active power only due to the high cost of the required inverter and transformer to supply reactive power by CPV system.

Static analysis is applied to investigate the impact of CPV location on the voltage stability of network . AC contingency analysis is applied to network for CPV at different locations (bus 6,8 and 12). the voltage control mode is used to represent CPV for simualtion in this paper.

Fig. 4 shows the maximum voltage violation at the outage line (5-6) for IEEE-14 bus network with CPV at location bus 8. Also, as shown in Fig. 3 , the voltage drop in case CPV is greater than one without RE.In this case, synchronous condenser at bus 8 is replaced by CPV. So the voltage drop is increased as mentioned above.

From the ac contingency analysis, the worst contingency (single (2-3)) is found when the CPV is located at bus 8, 6 and 12. This event has severe impact on bus 3 as listed in Table 2 . 
From the above simulation results, the effect of the CPV on voltage stability of the network is positive when connected to bus 12 and 6 . These buses are near the loads and critical buses. The CPV serves these buses at any disturbance.

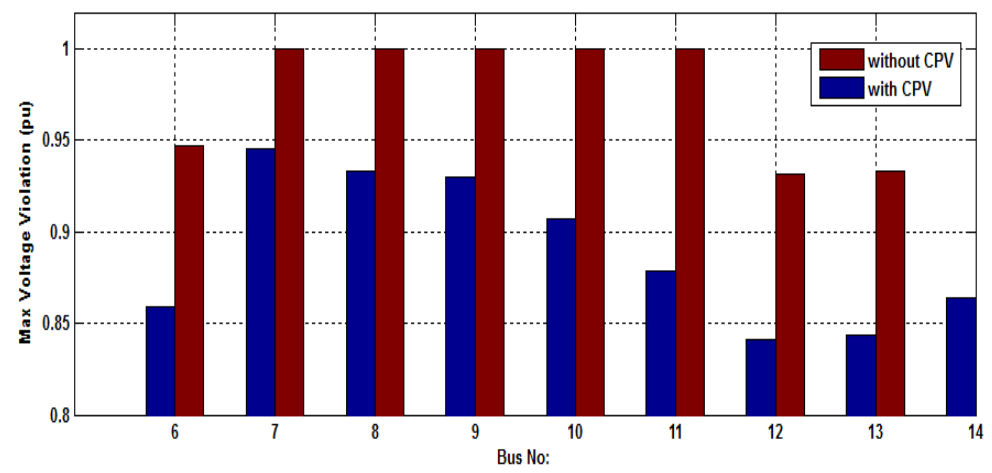

Fig. 4. Voltage buses at IEEE-14 with and without CPV at outage line (5-6).

Table 2. The impact of CPV location at outage line (2-3)

\begin{tabular}{llcc}
\hline Worst contingency & CPV located at bus 6 & CPV located at bus 12 & CPV located at bus 8 \\
\hline & Maximum Voltage violation for bus 3 \\
Outage line from bus 2 to bus 3 & 0.89177 & 0.87214 & 0.88021 \\
\hline
\end{tabular}

- IEEE-14 bus with distributed PV generators

In this case, the penetration level is divided into two PV, where the sizing of PV is 40MW for each one. The distributed PV generators are connected to bus 8 and 10 .

AC contingency analysis is applied toIEEE-14 network with distributed CPV at bus 8, 10. Results are recorded in Table 3. That indicates the voltage drop at bus 3 at the outage line 2-3 is less than in case IEEE-14 bus without RE network and also for the integration of CPV at different locations. From recorded results in Table 3, the voltage stability of the network, in this case, is improved and enhanced.

Hereby, the location of distributed CPV in the network has effective performance. Adequate analysis should be implemented for defining the best location of CPV plants.

Table 3. Worst contingency for distributed PV at bus 8 and 10

\begin{tabular}{ccc}
\hline Worst Contingency & Bus No: & Max Voltage Violation(pu) \\
\hline Single 2-3 (1) & 3 & 0.89850 \\
Single 5-6 (1) & 12 & 0.93658 \\
Single 5-6 (1) & 13 & 0.93773 \\
\hline
\end{tabular}

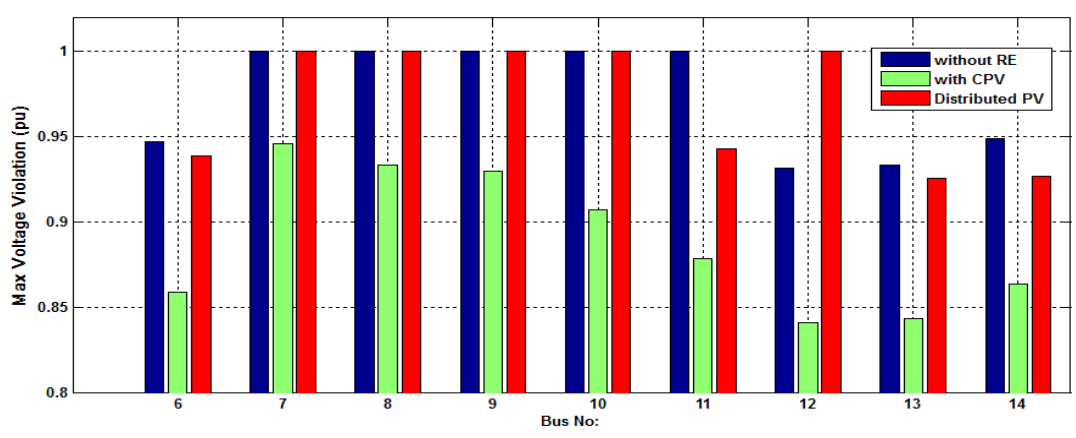

Fig. 5. Voltage violation buses for three cases.

To summarize the above results, Fig. 5 is developed to show the voltage buses at outage line (5-6) at 
each case and Table 4 listed these results. From this figure, the distributed PV generators that connected at bus 6,8 and 12 serves in voltage stability of the network. The penetration level of CPV is distributed at selected three buses. These buses are selected based on the ac contingency analysis tool.

Table 4. Voltage buses at worst contingencies for all cases

\begin{tabular}{lllll}
\hline Worst contingency & Bus N0: & IEEE-14 Without CPV & IEEE-14 with PV at bus 6,12,8 & IEEE-14 With CPV \\
\hline Single 2-3(1) & 3 & 0.89516 & 0.87966 & 0.88021 \\
Single 5-6(1) & 6 & 0.94721 & 0.93872 & 0.85893 \\
Single5-6(1) & 7 & 1 & 1 & 0.94549 \\
Single5-6(1) & 8 & 1 & 1 & 0.93343 \\
Single5-6(1) & 9 & 1 & 1 & 0.92995 \\
Single5-6(1) & 10 & 1 & 1 & 0.90696 \\
Single5-6(1) & 11 & 1 & 0.94250 & 0.87834 \\
Single5-6(1) & 12 & 0.93158 & 1 & 0.84107 \\
Single5-6(1) & 13 & 0.93318 & 0.92531 & 0.84328 \\
Single5-6(1) & 14 & 0.94881 & 0.92696 & 0.86369 \\
\hline
\end{tabular}

\section{Conclusion}

In this paper, the integration of PV in to grid with a large scale is demonstrated and discussed in details. The CPV and distributed CPV are used to show the impact on the voltage stability.

Unlike other studies, the selection of location of CPV is determined as an initial step according to location impact on grid stability. Ac contingency is used to monitor the voltage buses at certain events and define the critical loading bus/ events.

From the numerical results, the distributed CPV improves the voltage stability of the network, rather than centralized CPV. Distributed CPV connects at different locations and near to the load. Therefore, the impact of distributed CPV is minimized with respect to voltage stability issue.

In case of large scale integrated CPV plant, contingency analysis should be implemented prior of site /bus selection, to define CPV integration impact on the whole grid stability. Integration location/bus, in this case, has the same importance of other familiar factors as irradiance and land size in CPV plant planning.

\section{Acknowledgements}

The authors gratefully acknowledge the grant and support of "Small scale thermal solar district units for Mediterranean communities", Priority 2 - Topic 2.3 Solar Energy, Ref. I-A/2.3/174, ENPI CBCMED. The research leading to these results has received funding from the European Union with Academy of Scientific Research and Technology.

\section{References}

[1] IEA. Archived from the original on 7 October 2014. Technology roadmap: solar photovoltaic energy. (2014). [Online]. Available: http://www.iea.org

[2] Andrews RW, Andrew P, Joshua MP. Photovoltaic system performance enhancement with non-tracking planar concentrators: experimental results and BDRF based modelling. In: Proc. IEEE 39th Photovoltaic Specialists Conference, June 16-21, 2013:0229-0234.

[3] Amin AM, Mahfouz MMA, Youssef EB. Improvement the integration of zafarana wind farm connected to egyptian unified power grid. In: Proc. 46 International Universities' Power Engineering Conference, Soest, Germany, September 5-8, 2011.

[4] Amin M, Mahfouz MMA, Youssef EB. Dynamic disturbance of wind farm connected to a14-bus network. In: Proc.

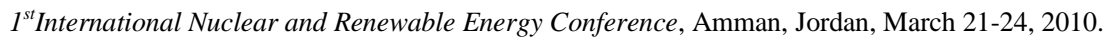

[5] Power System Voltage Stability. McGraw-Hill; 1994.

[6] Trends in Photovoltaic Applications, Survey Report of Selected IEA Countries Between (1992-2009). International Energy Agency, Photovoltaic Power Systems Program. (August 2010). [Online]. Available: 
http://www.ieapvps.org/index.php?id=92\&eID=dam_frontend_push\&docID=432M

[7] Ayoub J, Dignard-Bailey L, Poissant Y. (June 2010). National Survey report of PV power applications in Canada 2009. International Energy Agency, Photovoltaic Power Systems Program. [Online]. Available: http://www.ieapvps

[8] Eltawil, Zhao Z. Grid-connected photovoltaic power systems: technical and potential problems-A review. Renewable and Sustainable Energy Review, 2010; 14(1):112-129.

[9] Wang YB, Wu CS, Liao H, Xu HH. Study on impacts of large-scale photovoltaic power station on power grid voltage profile. In: Proc. Third International Conference on Electric Utility Deregulation, Restructuring and Power Technologies, 2008.

[10] IEEE Application Guide for IEEE Std 1547, IEEE Standard for Interconnecting Distributed Resources with Electric Power Systems, IEEE Std 1547.2-2008, 2009:1-207.

[11] Thompson JR, McConell RD, Mosleh M. Cost analysis of a concentrator photovoltaic hydrogen production system. In: Proc. International Conference of Solar Concentration for the Generation of Electricity or Hydrogen, A. Scottsdale, Arizona, 1-5 May 2005.

[12] International Grid Code Comparison. [Online]. Available: http://www.glgroup.com/pdf/IGCC_list.pdf

[13] Tamimi B, Cañizares C, Bhattacharya K. Modeling and performance analysis of large solar photo-voltaic generation on voltage stability and inter-area oscillations. In: Proc. IEEE PES General Meeting, July 2011.

[14] "Modeling new forms of generation and storage," CIGRE technical brochure, Nov. 2000.

[15] Ueda Y, Suzuki S, Ito T. Grid stabilization by use of an energy storage system for a large-scale PV generation plant. ECS Transactions, Oct. 2009; 16(34):17-25.

[16] PSS®E 33.2 Program Operation Manual, Siemens Power Technologies.

[17] [Online]. Available: http://www.power.uwaterloo.ca/ fmilano

[18] Youssef E, El Azab RM, Amin AM. Comparative study of voltage stability analysis for renewable energy grid-connected systems using PSS/E. In: Proc. IEEE Southeastc on Conference, 2015. 